\title{
An Extreme Problem for a Volterra Type Integral Inclusion
}

\author{
M. A. Sadygov \\ Baku State University, Baku, Azerbaijan \\ Email: mis reddin08@rambler.ru
}

How to cite this paper: Sadygov, M.A. (2019) An Extreme Problem for a Volterra Type Integral Inclusion. Open Access Library Journal, 6: e5605.

https://doi.org/10.4236/oalib.1105605

Received: July 16, 2019

Accepted: July 30, 2019

Published: August 2, 2019

Copyright $\odot 2019$ by author(s) and Open Access Library Inc.

This work is licensed under the Creative Commons Attribution International License (CC BY 4.0).

http://creativecommons.org/licenses/by/4.0/

\begin{abstract}
In the work, we have studied the dependencies of the solutions to integral inclusions from perturbation and investigated an extremal problem for integral inclusions. We obtained necessary and sufficient minimum conditions for extremal problems of Volterra type convex inclusions. We also studied a nonconvex extremal problem for the Volterra type inclusion. We obtained a high order necessary condition in the extremal problem for the Volterra type inclusion.
\end{abstract}

\section{Subject Areas}

Mathematical Analysis

\section{Keywords}

Integral Functional, Normal Integrant, Volterra Type Inclusion, The Multivalued Mapping

\section{Dependence of the Solution to the Integral Inclusion from Perturbation}

Let $R^{n}$ be the $n$-dimensional Euclidean space. The set of all nonempty compact (convex compact) subsets in $R^{n}$ we will designate as $\operatorname{comp} R^{n}\left(\operatorname{conv} R^{n}\right)$; $k:\left[t_{0}, T\right]^{2} \rightarrow M_{n}$ is the continuous matrix function, wherewith $M_{n}$ being the set of all square $n \times n$ matrices of real elements $\left(b_{i j}\right) ; z:\left[t_{0}, T\right] \rightarrow R^{n}$ the continuous function; $F:\left[t_{0}, T\right] \times R^{n} \rightarrow \operatorname{comp} R^{n}$ the setvalued mapping.

Assume that if a vector is multiplied by a matrix, then the vector is a row vector, if a matrix is multiplied by a vector, then the vector is a column vector.

Let us consider a problem for inclusion

$$
u(t) \in F\left(t, \int_{t_{0}}^{t} k(t, s) u(s) \mathrm{d} s+z(t)\right)
$$


The function $u(\cdot) \in L_{1}^{n}\left[t_{0}, T\right]$ satisfying (1) we will call the solution to problem (1) (see [1]).

Let $a=\max _{t, s \in\left[t_{0}, T\right]}\|k(t, s)\|=\max _{t, s \in\left[t_{0}, T\right]} \sum_{i=1}^{n} \sum_{j=1}^{n}\left|k_{i, j}(t, s)\right|$, if $k:\left[t_{0}, T\right]^{2} \rightarrow M_{n}$ is the continuous matrix function.

Theorem 1. Let $k:\left[t_{0}, T\right]^{2} \rightarrow M_{n}$ be the continuous matrix function, $z:\left[t_{0}, T\right] \rightarrow R^{n}$ the continuous function, $F:\left[t_{0}, T\right] \times R^{n} \rightarrow \operatorname{compR}^{n}$ the multivalued mapping, $t \rightarrow F(t, x)$ is measurable on $t$, and there exists a summable function $M(t)>0$ such that $\rho_{x}\left(F(t, x), F\left(t, x_{1}\right)\right) \leq M(t)\left|x-x_{1}\right|$ for $x, x_{1} \in R^{n}$. Moreover, let $\rho(\cdot) \in L_{1}\left[t_{0}, T\right]$ and $\bar{u}(\cdot) \in L_{1}^{n}\left[t_{0}, T\right]$ be such that $d\left(\bar{u}(t), F\left(t, \int_{t_{0}}^{t} k(t, s) \bar{u}(s) \mathrm{d} s+z(t)\right)\right) \leq \rho(t)$ for $t \in\left[t_{0}, T\right]$. Then there exists such a solution $u(\cdot) \in L_{1}^{n}\left[t_{0}, T\right]$ to problem (1) that

$$
\begin{gathered}
\left|\int_{t_{0}}^{t} k(t, s) u(s) \mathrm{d} s-\int_{t_{0}}^{t} k(t, s) \bar{u}(s) \mathrm{d} s\right| \leq a \int_{t_{0}}^{t} \mathrm{e}^{m(t)-m(s)} \rho(s) \mathrm{d} s, \\
|u(t)-\bar{u}(t)| \leq \rho(t)+a M(t) \int_{t_{0}}^{t} \mathrm{e}^{m(t)-m(s)} \rho(s) \mathrm{d} s
\end{gathered}
$$

for $t \in\left[t_{0}, T\right]$, where $m(t)=a \int_{t_{0}}^{t} M(s) \mathrm{d} s$.

\section{On Subdifferential of the Integral Functional}

Let $f:\left[t_{0}, T\right] \times R^{n} \rightarrow(-\infty,+\infty]$ is the normal convex integrant (see [2]).

Let consider a subdifferential of the integral functional

$$
J(u(\cdot))=\int_{t_{0}}^{T} f\left(t, \int_{t_{0}}^{t} k(t, s) u(s) \mathrm{d} s+z(t)\right) \mathrm{d} t
$$

in $L_{1}^{n}\left[t_{0}, T\right]$.

Theorem 2. If $k:\left[t_{0}, T\right]^{2} \rightarrow M_{n}$ be the continuous matrix function, $z:\left[t_{0}, T\right] \rightarrow R^{n}$ the continuous function, $f:\left[t_{0}, T\right] \times R^{n} \rightarrow(-\infty,+\infty]$ is the normal convex integrant and function $f\left(t, \int_{t_{0}}^{t} k(t, s) \bar{u}(s) \mathrm{d} s+z(t)+x\right)$ is summable for $x \in R^{n},|x| \leq \delta$, where $\bar{u}(\cdot) \in L_{1}^{n}\left[t_{0}, T\right]$, then $\partial J(\bar{u}(\cdot))$ is nonempty and $v^{*} \in L_{\infty}^{n}\left[t_{0}, T\right]$ belongs to $\partial J(\bar{u}(\cdot))$ if and only if, there exist $u^{*}(\cdot) \in L_{1}^{n}\left[t_{0}, T\right], \quad u^{*}(t) \in \partial f\left(t, \int_{t_{0}}^{t} k(t, s) \bar{u}(s) \mathrm{d} s+z(t)\right)$, such, that $v^{*}(s)=\int_{s}^{T} k(t, s)^{t} u^{*}(t) \mathrm{d} t$, where $k(\tau, t)^{t}$ is the transpose of the matrix $k(\tau, t)$.

Theorem 3. If $k:\left[t_{0}, T\right]^{2} \rightarrow M_{n}$ be measurable bounded matrix function, $z:\left[t_{0}, T\right] \rightarrow R^{n}$ be measurable bounded function, $f:\left[t_{0}, T\right] \times R^{n} \rightarrow(-\infty,+\infty]$ is the normal convex integrant and function $f\left(t, \int_{t_{0}}^{t} k(t, s) \bar{u}(s) \mathrm{d} s+z(t)+x\right)$ is summable for $x \in R^{n},|x| \leq \delta$, where $\bar{u}(\cdot) \in L_{1}^{n}\left[t_{0}, T\right]$, then $\partial J(\bar{u}(\cdot))$ is nonempty and functional $v^{*} \in L_{\infty}^{n}\left[t_{0}, T\right]$ belongs to $\partial J(\bar{u}(\cdot))$ if and only if, there exist $u^{*}(\cdot) \in L_{1}^{n}\left[t_{0}, T\right], \quad u^{*}(t) \in \partial f\left(t, \int_{t_{0}}^{t} k(t, s) \bar{u}(s) \mathrm{d} s+z(t)\right)$, such, that $v^{*}(s)=\int_{s}^{T} k(t, s)^{t} u^{*}(t) \mathrm{d} t$. 


\section{On Subdifferential of the Terminal Functional}

Let $k:\left[t_{0}, T\right]^{2} \rightarrow M_{n}$ continuous matrix function, $z:\left[t_{0}, T\right] \rightarrow R^{n}$ continuous function, $\varphi: R^{n} \rightarrow(-\infty,+\infty]$ proper convex function in $R^{n}$. Consider a subdifferential of the terminal functional $F(u(\cdot))=\varphi\left(\int_{t_{0}}^{T} k(T, s) u(s) \mathrm{d} s+z(T)\right)$ in $L_{1}^{n}\left[t_{0}, T\right]$, where $z(\cdot) \in C^{n}\left[t_{0}, T\right]$.

Theorem 4. If $\varphi$-proper convex function in $R^{n}$ and continuous in the point $\int_{t_{0}}^{T} k(T, s) \bar{u}(s) \mathrm{d} s+z(T)$, then

$$
\partial F(\bar{u}(\cdot))=\left\{b k(T, s): b \in \partial \varphi\left(\int_{t_{0}}^{T} k(T, s) \bar{u}(s) \mathrm{d} s+z(T)\right)\right\} .
$$

\section{Convex Extremal Problem for Integral Inclusions}

Let $k:\left[t_{0}, T\right]^{2} \rightarrow M_{n}$ be the continuous matrix function, $z:\left[t_{0}, T\right] \rightarrow R^{n}$ the continuous function. Hereafter we will assume that $f:\left[t_{0}, T\right] \times R^{n} \rightarrow(-\infty,+\infty]$ is the normal convex integrant, $\varphi: R^{n} \rightarrow(-\infty,+\infty]$ the convex function. Let $t_{0}<T, F:\left[t_{0}, T\right] \times R^{n} \rightarrow \operatorname{comp} R^{n} \cup\{\varnothing\} \quad$ is the multivalued mapping.

The problem of minimization of the functional

$$
J(u)=\varphi\left(\int_{t_{0}}^{T} k(T, s) u(s) \mathrm{d} s+z(T)\right)+\int_{t_{0}}^{T} f\left(t, \int_{t_{0}}^{t} k(t, s) u(s) \mathrm{d} s+z(t)\right) \mathrm{d} t
$$

is considered under the following constraints

$$
u(t) \in F\left(t, \int_{t_{0}}^{t} k(t, s) u(s) \mathrm{d} s+z(t)\right),
$$

where $t \in\left[t_{0}, T\right], u(\cdot) \in L_{1}^{n}\left[t_{0}, T\right]$.

Introducing the notation $\omega(t, x, z)=\left\{\begin{array}{ll}0, & z \in F(t, x) \\ +\infty, & z \notin F(t, x)\end{array}\right.$ we have that problem (2) and (3) is equivalent to the minimization of the functional

$$
\begin{aligned}
J_{1}(u)= & \varphi\left(\int_{t_{0}}^{T} k(T, s) u(s) \mathrm{d} s+z(T)\right)+\int_{t_{0}}^{T} f\left(t, \int_{t_{0}}^{t} k(t, s) u(s) \mathrm{d} s+z(t)\right) \mathrm{d} t \\
& +\int_{t_{0}}^{T} \omega\left(t, \int_{t_{0}}^{t} k(t, s) u(s) \mathrm{d} s+z(t), u(t)\right) \mathrm{d} t
\end{aligned}
$$

among all functions $u(\cdot) \in L_{1}^{n}\left[t_{0}, T\right]$.

Let the mapping $t \rightarrow g r F_{t}=\{(x, y): y \in F(t, x)\}$ be measurable on $\left[t_{0}, T\right]$, the set $g r F_{t}$ be closed and convex for almost all $t \in\left[t_{0}, T\right]$ and $F(t, x)$ be compact for all $(t, x)$. From here it follows that $\omega(t, x, z)$ is a convex normal integrant on $\left[t_{0}, T\right] \times\left(R^{n} \times R^{n}\right)$.

Let us consider the following functional

$$
\begin{aligned}
S(u, v)= & \varphi\left(\int_{t_{0}}^{T} k(T, s) u(s) \mathrm{d} s+z(T)\right)+\int_{t_{0}}^{T} f\left(t, \int_{t_{0}}^{t} k(t, s) u(s) \mathrm{d} s+z(t)\right) \mathrm{d} t \\
& +\int_{t_{0}}^{T} \omega\left(t, \int_{t_{0}}^{t} k(t, s) u(s) \mathrm{d} s+z(t), u(t)+v(t)\right) \mathrm{d} t,
\end{aligned}
$$

where $v(\cdot) \in L_{1}^{n}\left[t_{0}, T\right]$. Let $h(v)=\inf _{\left.u \in L_{L}^{n} t_{0}, T\right]} S(u, v)$. The problem (2) and (3) is called stable, if $h(0)$ is finite and function $h$ is subdifferentiable at zero (see [3]).

Lemma 1. Let $F:\left[t_{0}, T\right] \times R^{n} \rightarrow \operatorname{comp} R^{n} \cup\{\varnothing\}$; the mapping $t \rightarrow F(t, x)$ 
be measurable on $\left[t_{0}, T\right]$; the mapping $x \rightarrow F(t, x)$ be closed and convex for almost all $t \in\left[t_{0}, T\right]$, i.e. $g r F_{t}$ be closed and convex for almost all $t \in\left[t_{0}, T\right]$; there exist such a summable function $\lambda(t)$ that $\|F(t, x)\| \leq \lambda(t)(1+|x|)$ for $x \in R^{n}$; there exist a solution $u_{0}(t)$ to the problem

$u_{0}(t) \in F\left(t, \int_{t_{0}}^{t} k(t, s) u_{0}(s) \mathrm{d} s+z(t)\right)$ such that $x_{0}(t)=\int_{t_{0}}^{t} k(t, s) u_{0}(s) \mathrm{d} s+z(t)$ belongs to $\operatorname{dom} F_{t}=\{x: F(t, x) \neq \varnothing\}$ coupled with some $\varepsilon$ tube, i.e. $\left\{x:\left|x_{0}(t)-x\right| \leq \varepsilon\right\} \subset \operatorname{dom}_{t} ; f:\left[t_{0}, T\right] \times R^{n} \rightarrow(-\infty,+\infty]$ the normal convex integrant; $\varphi: R^{n} \rightarrow(-\infty,+\infty]$ the convex function and $\inf _{u \in L_{1}^{n}\left[t_{0}, T\right]} J_{1}(u)$ is finite; the function $f\left(t, \int_{t_{0}}^{t} k(t, s) u_{0}(s) \mathrm{d} s+z(t)+y\right)$ be summarized for $y \in R^{n}$, $|y|<r$, where $r>0$, and function $\varphi(\cdot)$ be continuous at the point $\int_{t_{0}}^{T} k(T, s) u_{0}(s) \mathrm{d} s+z(T)$. Then the function $h$ is subdifferentiable at zero, i.e. problem (2) and (3) is stable.

Let $v \in R^{n}$. Assume

$$
\omega^{0}(t, x, v)=\inf _{z \in R^{n}}\{(z \mid v)+\omega(t, x, z)\}=\inf \{(z \mid v): z \in F(t, x)\},
$$

where $\inf \varnothing=+\infty$.

Theorem 5. Let $F:\left[t_{0}, T\right] \times R^{n} \rightarrow \operatorname{comp} R^{n} \cup\{\varnothing\}$; the mapping $t \rightarrow F(t, x)$ be measurable on $\left[t_{0}, T\right]$; the mapping $x \rightarrow F(t, x)$ be closed and convex for almost all $t \in\left[t_{0}, T\right] ; f$ be the normal convex integrant on $\left[t_{0}, T\right] \times R^{n} ; \varphi$ the convex function on $R^{n} ; k:\left[t_{0}, T\right]^{2} \rightarrow M_{n}$ the continuous matrix function; $z:\left[t_{0}, T\right] \rightarrow R^{n}$ the continuous function. For the function $\bar{u}(\cdot) \in L_{1}^{n}\left[t_{0}, T\right]$ to minimize the functional (2) among all the solutions to the problem (3), it is sufficient that there exist $u_{1}^{*}(\cdot), u_{2}^{*}(\cdot) \in L_{1}^{n}\left[t_{0}, T\right]$ and $b \in R^{n}$ such that

1) $u_{1}^{*}(t) \in \partial f\left(t, \int_{t_{0}}^{t} k(t, s) \bar{u}(s) \mathrm{d} s+z(t)\right)$

2) $b \in \partial \varphi\left(z(T)+\int_{t_{0}}^{T} k(T, s) \bar{u}(s) \mathrm{d} s\right)$,

3)

$$
\begin{aligned}
& u_{2}^{*}(t) \in \partial \omega^{0}\left(t, \int_{t_{0}}^{t} k(t, s) \bar{u}(s) \mathrm{d} s+z(t), \int_{t}^{T} k(\tau, t)^{t}\left(u_{1}^{*}(\tau)+u_{2}^{*}(\tau)\right) \mathrm{d} \tau+K(T, t)^{t} b\right), \\
& \omega^{0}\left(t, z(t)+\int_{t_{0}}^{t} k(t, s) \bar{u}(s) \mathrm{d} s, \int_{t}^{T} k(\tau, t)^{t}\left(u_{1}^{*}(\tau)+u_{2}^{*}(\tau)\right) \mathrm{d} \tau+K(T, t)^{t} b\right) \\
& 4)=\left(\bar{u}(t) \mid \int_{t}^{T} k(\tau, t)^{t}\left(u_{1}^{*}(\tau)+u_{2}^{*}(\tau)\right) \mathrm{d} \tau+K(T, t)^{t} b\right) \\
& +\omega\left(t, z(t)+\int_{t_{0}}^{t} k(t, s) \bar{u}(s) \mathrm{d} s, \bar{u}(t)\right),
\end{aligned}
$$

and if for $u_{0}(t)=\bar{u}(t)$ the condition of lemma 1 is satisfied, then conditions 1) - 4) become necessary.

\section{Nonconvex Extremal Problem for Integral Inclusions}

Let $k:\left[t_{0}, T\right]^{2} \rightarrow M_{n}$ be the continuous matrix function; $z:\left[t_{0}, T\right] \rightarrow R^{n}$ the 
continuous function, i.e. $z(\cdot) \in C^{n}\left[t_{0}, T\right]$. Hereafter we will assume that $f:\left[t_{0}, T\right] \times R^{n} \times R^{n} \rightarrow(-\infty,+\infty]$ is the normal integrant and $\varphi: R^{n} \rightarrow(-\infty,+\infty]$ is the function. Let $t_{0}<T, F:\left[t_{0}, T\right] \times R^{n} \rightarrow \operatorname{comp} R^{n}$ be the multivalued mapping.

We consider the following problem of minimization of the functional

$$
J(u)=\varphi\left(\int_{t_{0}}^{T} k(T, s) u(s) \mathrm{d} s+z(T)\right)+\int_{t_{0}}^{T} f\left(t, \int_{t_{0}}^{t} k(t, s) u(s) \mathrm{d} s+z(t), u(t)\right) \mathrm{d} t,
$$

under the following constraints

$$
u(t) \in F\left(t, \int_{t_{0}}^{t} k(t, s) u(s) \mathrm{d} s+z(t)\right),
$$

where $t \in\left[t_{0}, T\right], u(\cdot) \in L_{1}^{n}\left[t_{0}, T\right]$.

Let $\psi(s, x, y)=\inf \{|z-y|: z \in F(s, x)\}$ and consider the minimization of the functional

$$
\begin{aligned}
J_{r}(u)= & \varphi\left(\int_{t_{0}}^{T} k(T, s) u(s) \mathrm{d} s+z(T)\right)+\int_{t_{0}}^{T} f\left(t, \int_{t_{0}}^{t} k(t, s) u(s) \mathrm{d} s+z(t), u(t)\right) \mathrm{d} t \\
& +r \int_{t_{0}}^{T} \psi\left(t, \int_{t_{0}}^{t} k(t, s) u(s) \mathrm{d} s+z(t), u(t)\right) \mathrm{d} t
\end{aligned}
$$

among all the functions $u(\cdot) \in L_{1}^{n}\left[t_{0}, T\right]$.

Theorem 6. If $\bar{u}(\cdot) \in L_{1}^{n}\left[t_{0}, T\right]$ is the solution to the problem (4) and (5), $F:\left[t_{0}, T\right] \times R^{n} \rightarrow \operatorname{comp} R^{n} \cup\{\varnothing\}$ and $t \rightarrow F(t, x)$ are measurable on $t$, $\bar{x}(t)=\int_{t_{0}}^{t} k(t, s) \bar{u}(s) \mathrm{d} s+z(t)$, there exist $k(\cdot) \in L_{1}\left[t_{0}, T\right], M(\cdot) \in L_{1}\left[t_{0}, T\right]$, $k_{1}>0, k_{2}>0$ and $\alpha>0$ such that

$$
\begin{gathered}
B(\bar{x}(t), \alpha) \subset \operatorname{dom}_{t}=\left\{x \in R^{n}: F(t, x) \neq \varnothing\right\} \text { at } t \in\left[t_{0}, T\right] \text { and } \\
|\varphi(z)-\varphi(u)| \leq k_{2}|z-u|, \\
\left|f\left(t, x_{1}, y_{1}\right)-f\left(t, x_{2}, y_{2}\right)\right| \leq k(t)\left|x_{1}-x_{2}\right|+k_{1}\left|y_{1}-y_{2}\right|, \\
\rho_{X}\left(F\left(t, x_{1}\right), F\left(t, x_{2}\right)\right) \leq M(t)\left|x_{1}-x_{2}\right|
\end{gathered}
$$

for $z, u \in B(\bar{x}(T), \alpha), \quad x_{1}, x_{2} \in B(\bar{x}(t), \alpha), \quad y_{1}, y_{2} \in R^{n}$. Then there exist a number $r_{0}>0$ such that $\bar{u}(t)$ minimizes the functional $J_{r}(u)$ in $D$ for $r \geq r_{0}$, where $D=\left\{u(\cdot) \in L_{1}^{n}\left[t_{0}, T\right]:\|u(\cdot)-\bar{u}(\cdot)\|_{L_{1}^{n}\left[t_{0}, T\right]} \leq \frac{\alpha}{\beta}\right\}$, $\beta>\left(1+a\left(\mathrm{e}^{m(T)}+a \mathrm{e}^{m(T)} \int_{t_{0}}^{T} M(t) \mathrm{d} t\right)\right)\left(a \int_{t_{0}}^{T} M(t) \mathrm{d} t+1\right)+a, \quad m(t)=a \int_{t_{0}}^{t} M(s) \mathrm{d} s$.

Theorem 7. Let the condition of the theorem 6 be satisfied and the function $\bar{u}(t)$ among all solutions to the problem (5) minimizes the functional (4). Then there exists $u^{*}(\cdot) \in L_{1}^{n}\left[t_{0}, T\right]$ and $b \in R^{n}$ such that

$$
\begin{array}{r}
\left(u^{*}(t),-\int_{t}^{T} k(\tau, t)^{t} u^{*}(\tau) \mathrm{d} \tau-K(T, t)^{t} b\right) \\
1) \in \partial_{C}\left(f\left(t, \int_{t_{0}}^{t} k(t, s) \bar{u}(s) \mathrm{d} s+z(t), \bar{u}(t)\right)\right. \\
\left.+r \psi\left(t, \int_{t_{0}}^{t} k(t, s) \bar{u}(s) \mathrm{d} s+z(t), \bar{u}(t)\right)\right),
\end{array}
$$


2) $b \in \partial_{C} \varphi\left(z(T)+\int_{t_{0}}^{T} k(T, s) \bar{u}(s) \mathrm{d} s\right)$,

where $\partial_{C} g(\bar{x})$ is Clarke subdifferential of the function $g$ at the point $\bar{x}$ (see [4]).

\section{A Higher Order Necessary Condition in the Extremal Problem for the Volterra Type Inclusion}

Consider the problem (4) and (5), where $f(t, x, y)=f(t, x)$. Assume

$$
\psi(s, x, y)=\inf \{|z-y|: z \in F(s, x)\} .
$$

We consider the following problem of minimization of the function

$$
\begin{aligned}
J_{r}(u)= & \varphi\left(\int_{t_{0}}^{T} k(T, s) u(s) \mathrm{d} s+z(T)\right)+\int_{t_{0}}^{T} f\left(t, \int_{t_{0}}^{t} k(t, s) u(s) \mathrm{d} s+z(t)\right) \mathrm{d} t \\
& +r\left(\left(\int_{t_{0}}^{T} \psi\left(t, \int_{t_{0}}^{t} k(t, s) u(s) \mathrm{d} s+z(t), u(t)\right) \mathrm{d} t\right)^{\beta}\right. \\
& \left.+\|\bar{u}(\cdot)-u(\cdot)\|_{L_{1}^{n}}^{\beta-v}\left(\int_{t_{0}}^{T} \psi\left(t, \int_{t_{0}}^{t} k(t, s) u(s) \mathrm{d} s+z(t), u(t)\right) \mathrm{d} t\right)^{v}\right)
\end{aligned}
$$

among all functions $u(\cdot) \in L_{1}^{n}\left[t_{0}, T\right]$.

Let $\bar{u}(\cdot) \in L_{1}^{n}\left[t_{0}, T\right]$ be the solution to the problem (4) and (5). Let $\bar{x}(t)=\int_{t_{0}}^{t} k(t, s) \bar{u}(s) \mathrm{d} s+z(t)$.

Theorem 8. Let $F:\left[t_{0}, T\right] \times R^{n} \rightarrow \operatorname{comp}^{n}$ be the multivalued mapping, the mapping $t \rightarrow F(t, x)$ be measurable on $\left[t_{0}, T\right]$; $f$ be the normal integrant on $\left[t_{0}, T\right] \times R^{n} ; \varphi$ the function in $R^{n} ; k:\left[t_{0}, T\right]^{2} \rightarrow M_{n}$ the continuous matrix function; $z:\left[t_{0}, T\right] \rightarrow R^{n}$ the continuous function, and there exists a summable function $M(t)>0$ such that $\rho_{x}\left(F(t, x), F\left(t, x_{1}\right)\right) \leq M(t)\left|x-x_{1}\right|$ for $x, x_{1} \in R^{n}$; there exist $k_{1}(\cdot) \in L_{1}\left[t_{0}, T\right], k_{1}(t)>0$ and number $k_{2}>0$ such that

$$
\left|f\left(t, x_{1}\right)-f\left(t, x_{2}\right)\right| \leq k_{1}(t)\left|x_{1}-x_{2}\right|^{v}\left(\left|x_{2}-\bar{x}(t)\right|^{\beta-v}+\left|x_{1}-x_{2}\right|^{\beta-v}\right)
$$

for $x_{1}, x_{2} \in R^{n}$,

$$
|\varphi(x)-\varphi(y)| \leq k_{2}|x-y|^{\nu}\left(|y-\bar{x}(T)|^{\beta-v}+|x-y|^{\beta-v}\right)
$$

for $x, y \in R^{n}$ (see [5]). If the function $\bar{u}(t)$ among all solutions of the problem (5) minimizes the functional (4), then there exists a number $r_{0}>0$ such that $\bar{u}(t)$ minimizes the functional $J_{r}(u)$ in $L_{1}^{n}\left[t_{0}, T\right]$ for $r \geq r_{0}$.

Let $g:\left[t_{0}, T\right] \times R^{n} \rightarrow(-\infty,+\infty]$ be the normal integrant on $\left[t_{0}, T\right] \times R^{n}$; $e: R^{n} \rightarrow(-\infty,+\infty]$ the function.

Let assume

$$
S(u)=e\left(\int_{t_{0}}^{T} k(T, s) u(s) \mathrm{d} s+z(T)\right)+\int_{t_{0}}^{T} g\left(t, \int_{t_{0}}^{t} k(t, s) u(s) \mathrm{d} s+z(t)\right) \mathrm{d} t,
$$




$$
\begin{aligned}
H_{r}(u)= & J(u)-S(u)+r\left(\left(\int_{t_{0}}^{T} \psi\left(t, \int_{t_{0}}^{t} k(t, s) u(s) \mathrm{d} s+z(t), u(t)\right) \mathrm{d} t\right)^{\beta}\right. \\
& \left.+\|\bar{u}(\cdot)-u(\cdot)\|_{L_{1}^{n}}^{\beta-v}\left(\int_{t_{0}}^{T} \psi\left(t, \int_{t_{0}}^{t} k(t, s) u(s) \mathrm{d} s+z(t), u(t)\right) \mathrm{d} t\right)^{v}\right) \\
= & \varphi\left(\int_{t_{0}}^{T} k(T, s) u(s) \mathrm{d} s+z(T)\right)-e\left(\int_{t_{0}}^{T} k(T, s) u(s) \mathrm{d} s+z(T)\right) \\
& +\int_{t_{0}}^{T} f\left(t, \int_{t_{0}}^{t} k(t, s) u(s) \mathrm{d} s+z(t)\right) \mathrm{d} t-\int_{t_{0}}^{T} g\left(t, \int_{t_{0}}^{t} k(t, s) u(s) \mathrm{d} s+z(t)\right) \mathrm{d} t \\
& +r\left(\left(\int_{t_{0}}^{T} \psi\left(t, \int_{t_{0}}^{t} k(t, s) u(s) \mathrm{d} s+z(t), u(t)\right) \mathrm{d} t\right)^{\beta}\right. \\
& \left.+\|\bar{u}(\cdot)-u(\cdot)\|_{L_{1}^{n}}^{\beta-v}\left(\int_{t_{0}}^{T} \psi\left(t, \int_{t_{0}}^{t} k(t, s) u(s) \mathrm{d} s+z(t), u(t)\right) \mathrm{d} t\right)^{v}\right) .
\end{aligned}
$$

Theorem 9. Let $F:\left[t_{0}, T\right] \times R^{n} \rightarrow \operatorname{compR} R^{n}$ the multivalued mapping, the mapping $t \rightarrow F(t, x)$ be measurable on $\left[t_{0}, T\right]$; $f$ be the normal integrant on $\left[t_{0}, T\right] \times R^{n} ; \varphi$ the function in $R^{n} ; k:\left[t_{0}, T\right]^{2} \rightarrow M_{n}$ the continuous matrix function; $z:\left[t_{0}, T\right] \rightarrow R^{n}$ the continuous function and there exist a summable function $M(t)>0$ such that $\rho_{x}\left(F(t, x), F\left(t, x_{1}\right)\right) \leq M(t)\left|x-x_{1}\right|$ for $x, x_{1} \in R^{n}$; there exist the normal integrant $g:\left[t_{0}, T\right] \times R^{n} \rightarrow(-\infty,+\infty]$, the functions $e: R^{n} \rightarrow(-\infty,+\infty], \quad k_{1}(\cdot) \in L_{1}\left[t_{0}, T\right], k_{1}(t)>0$ and number $k_{2}>0$ such that

$$
\begin{aligned}
& \left|f\left(t, x_{1}\right)-g\left(t, x_{1}\right)-f\left(t, x_{2}\right)+g\left(t, x_{2}\right)\right| \\
& \leq k_{1}(t)\left|x_{1}-x_{2}\right|^{v}\left(\left|x_{2}-\bar{x}(t)\right|^{\beta-v}+\left|x_{1}-x_{2}\right|^{\beta-v}\right)
\end{aligned}
$$

for $x_{1}, x_{2} \in R^{n}$, where $\bar{x}(t)=\int_{t_{0}}^{t} k(t, s) \tilde{u}(s) \mathrm{d} s+z(t)$,

$$
|\varphi(x)-e(x)-\varphi(y)+e(y)| \leq k_{2}|x-y|^{v}\left(|y-\bar{x}(T)|^{\beta-v}+|x-y|^{\beta-v}\right)
$$

for $x, y \in R^{n}$ and let $\tilde{u}(\cdot) \in L_{1}^{n}\left[t_{0}, T\right]$ solutions of the problem (4)-(5). Then there exist a number $r_{0}>0$ such that $\tilde{u}(t)$ minimizes the functional $H_{r}(u)$ in $u \in\left\{v \in L_{1}^{n}\left[t_{0}, T\right]: S\left(w_{v}\right) \leq S(\tilde{u})\right\}$ for $r \geq r_{0}$, where $w_{v}$ solutions to the problem (5), which satisfy the main theorem 1 for $\bar{u}(\cdot)=v(t)$.

It's possible to get the local variant of theorems 8 and 9 analogical to theorem 6 .

Let assume

$$
\begin{aligned}
& J_{r}^{\{\beta\}+}(\bar{u} ; u)=\varlimsup_{\lambda \downarrow 0} \frac{1}{\lambda^{\beta}}\left(J_{r}(\bar{u}+\lambda u)-J_{r}(\bar{u})\right), \\
& J_{r}^{\{\beta\}-}(\bar{u} ; u)=\frac{\lim _{\lambda \downarrow 0}}{\lambda^{\beta}}\left(J_{r}(\bar{u}+\lambda u)-J_{r}(\bar{u})\right)
\end{aligned}
$$

for $u(\cdot) \in L_{1}^{n}\left[t_{0}, T\right]$.

Corollary 1 . If the condition of theorem 8 is satisfied, then there exist a number $r_{0}>0 \quad$ such that $J_{r}^{\{\beta\}+}(\bar{u} ; u) \geq J_{r}^{\{\beta\}-}(\bar{u} ; u) \geq 0 \quad$ for $\quad r \geq r_{0} \quad$ and $u(\cdot) \in L_{1}^{n}\left[t_{0}, T\right]$. 


\section{Let assume}

$$
\begin{aligned}
& E_{r}(u)=\varphi^{\{\beta\}-}\left(\int_{t_{0}}^{T} k(T, s) \bar{u}(s) \mathrm{d} s+z(T) ; \int_{t_{0}}^{T} k(T, s) u(s) \mathrm{d} s\right) \\
& +\int_{t_{0}}^{T} f^{\{\beta\}+}\left(t, \int_{t_{0}}^{t} k(t, s) \bar{u}(s) \mathrm{d} s+z(t) ; \int_{t_{0}}^{t} k(t, s) u(s) \mathrm{d} s\right) \mathrm{d} t \\
& +r\left(\left(\int_{t_{0}}^{T} \psi^{\{1\}+}\left(t, \int_{t_{0}}^{t} k(t, s) \bar{u}(s) \mathrm{d} s+z(t), \bar{u}(t) ; \int_{t_{0}}^{t} k(t, s) u(s) \mathrm{d} s, u(t)\right) \mathrm{d} t\right)^{\beta}\right. \\
& \left.+\|u(\cdot)\|_{L_{1}^{n}}^{\beta-v}\left(\int_{t_{0}}^{T} \psi^{\{1\}+}\left(t, \int_{t_{0}}^{t} k(t, s) \bar{u}(s) \mathrm{d} s+z(t), \bar{u}(t) ; \int_{t_{0}}^{t} k(t, s) u(s) \mathrm{d} s, u(t)\right) \mathrm{d} t\right)^{v}\right) .
\end{aligned}
$$

Theorem 10. If the condition of theorem 8 is satisfied, then there exist a number $r_{0}>0$ such that $E_{r}(u) \geq 0$ for $r \geq r_{0}$ and $u(\cdot) \in L_{1}^{n}\left[t_{0}, T\right]$.

\section{Conflicts of Interest}

The author declares no conflicts of interest regarding the publication of this paper.

\section{References}

[1] Sadygov, M.A. (2013) An Extremal Problem for Integral Inclusion. Preprint No. 1, Baku, 129 p.

[2] Rockafellar, R.T. and Wets, R.J.-B. (2009) Variational Analysis. Springer, Berlin, 734 p.

[3] Ekeland, I. and Temam, R. (1979) Convex Analysis and Variational Problems. Mir, Moscow, $309 \mathrm{p}$.

[4] Clarke, F. (2013) Functional Analysis, Calculus of Variations and Optimal Control. Springer-Verlag, London, 591 p. https://doi.org/10.1007/978-1-4471-4820-3_4

[5] Sadygov, M.A. (2014) Subdifferential of High Orders and Optimization. LAP Lambert Academic Publishing, Saarbrucken, 359 p. 